\title{
Chronic Exposure and the Risk of Building Antibiotic Resistance in Rural Communities of the Developing World
}

\author{
A Rashid ${ }^{1}$ and MS Coyne ${ }^{2 *}$ \\ ${ }^{1}$ Department of Environmental Sciences, Rawalpindi Pakistan \\ ${ }^{2}$ Department of Plant and Soil Sciences, USA
}

*Corresponding author: Mark Coyne, Professor of Soil Microbiology, Department of Plant and Soil Sciences, University of Kentucky, Lexington Kentucky USA.

Received Date: March 8, 2019

Published Date: March 22, 2019

\begin{abstract}
Antibiotic resistance is a global issue, not least the question if resistance is intrinsic, acquired, or adaptive. The current scenario demands investigations to detect resistance in environments from the urban/rural nexus. The complexity of antibiotic resistance in the environment is linked with the characteristics of recipient compartments in various environmental systems. Much research focuses on resistance in point sources (hotspots) of exposure, but after release the risk of antibiotics affecting humans is quite complex. Dispersion, dilution, and concentration reduction are part of the natural cycle required to examine the fate of antibiotics in the environment. Antibiotic residues at any stage threaten public health no matter if they cause acute exposure or because of much lower concentrations persisting in sediments, soils, irrigation waters, groundwater, aerosols, and produce where they may reflect chronic exposure. In either case, human exposure is undeniable with differential impact. In view of local constraints and unintended feedback we propose a research agenda to enhance understanding the future of exposure intensity and the fate and synergistic effect of antibiotics released into the environment in developing resistance. We advocate the need to investigate sub-lethal antibiotic concentrations and human populations aimed at limiting the acute and chronic public exposure to antibiotic residues and resistant microbes from soil and water among occupational groups and rural population inhabiting resource-limited settings.
\end{abstract}

Keywords: Epidemiology; Toxicity; Antibiotic reservoirs; Residue persistence

\section{Introduction}

The benefits of antibiotic use cannot be overemphasized; they certainly outweigh the harm associated with their side effects. However, when antibiotics are abused or overused, that harm increases manifold with no benefit.

Global antibiotic production for human use is a $\$ 40$ billion-ayear industry. Approximately 250 to 300 tons year of antibiotics are used in human medicine and a much greater amount of antibiotics are produced for livestock and aquaculture. To fulfill ever increasing human demands, huge amounts of antibiotics are produced worldwide, with projections for additionally expanded production in the future [1]. It is estimated that global antibiotic use will rise to 105,596 tons year $^{-1}$ in 2030 , with China the largest consumer followed by the US, Brazil, Mexico, and India [2].
This increase reflects their broadened use for treating genuine contamination identified with human well-being and welfare, and for advancing and developing disease aversion in domesticated animals. In particular, low- and middle-income countries showed increased antibiotic use between 2000 and 2015 (+39\%) which suggests an alarming projection for a $200 \%$ rise globally by 2030 [3].

\section{Off-label Prescription of Antibiotics}

Environmental exposure to antibiotics is increasingly unavoidable in developing countries. Antibiotic use is valuable for livestock survival and if their use were denied a shortage in food products originating from livestock would be expected. Unsanitary conditions at farmhouses exist in rural areas where antibiotics 
are used to keep animals from becoming ill. Disease risk can be mitigated by improving sanitary conditions for animals, but it would be expensive to raise animals without using antibiotics. However, antibiotic product meant for human or veterinary use applied for unproven indication, or blindly used to get desired results in a manner not specified according to product characteristics, is an offlabel use that can be regarded as unethical. Another off-label use, in the absence of an authentic prescription, occurs when antibiotics are sold to users who (depending on their own knowledge) indulge in self-medication - mostly on a wider scale to include animal husbandry such as poultry, livestock, and humans.

\section{Antibiotics Entering the Environment}

Antibiotics, like many pharmaceuticals, enter the environment by many routes: from human use via hospital discharge, local sewerage treatment plants, and leachates from landfills. Antibiotics can enter surface waters from sewerage treatment plants specifically, or they can be exchanged through surface runoff from treated animals [4].

Indiscriminate discharge of water effluents has threatening consequences for public health and the environment. Exposure to polluted water is the prime cause of mortality for an overwhelming number of people. Estimates from the UN indicate that the world's waterways receive 2 million tons of industrial, sewage, and agricultural waste each year [5]. The sewage muck (biosolids) used as a fertilizer in agricultural land areas can be the prime cause of ground water exposure to antibiotics [6].

Antibiotics may also enter the environment through manure use on soil and discharge by grazing domesticated animals [7]. Yang et al. [8] demonstrated the presence of antibiotic resistant bacteria in animal manures and an increase in antibiotic resistance of soil microbial populations exposed to such manure. Veterinary antibiotics can enter water bodies either specifically if treated animals enter surface water. Many antibiotics are inadequately ingested and not totally utilized in human and other large animals, especially mammals. Considerable antibiotic (30-90\%) is discharged in urine and feces shortly after their ingestion as either the parent compound or as residuals [9]. Humans are therefore potentially and continuously exposed to water or soil already having antibiotic concentrations at lethal or sub lethal levels, which could result in human health implications by increasing antibiotic resistance (Figure 1).

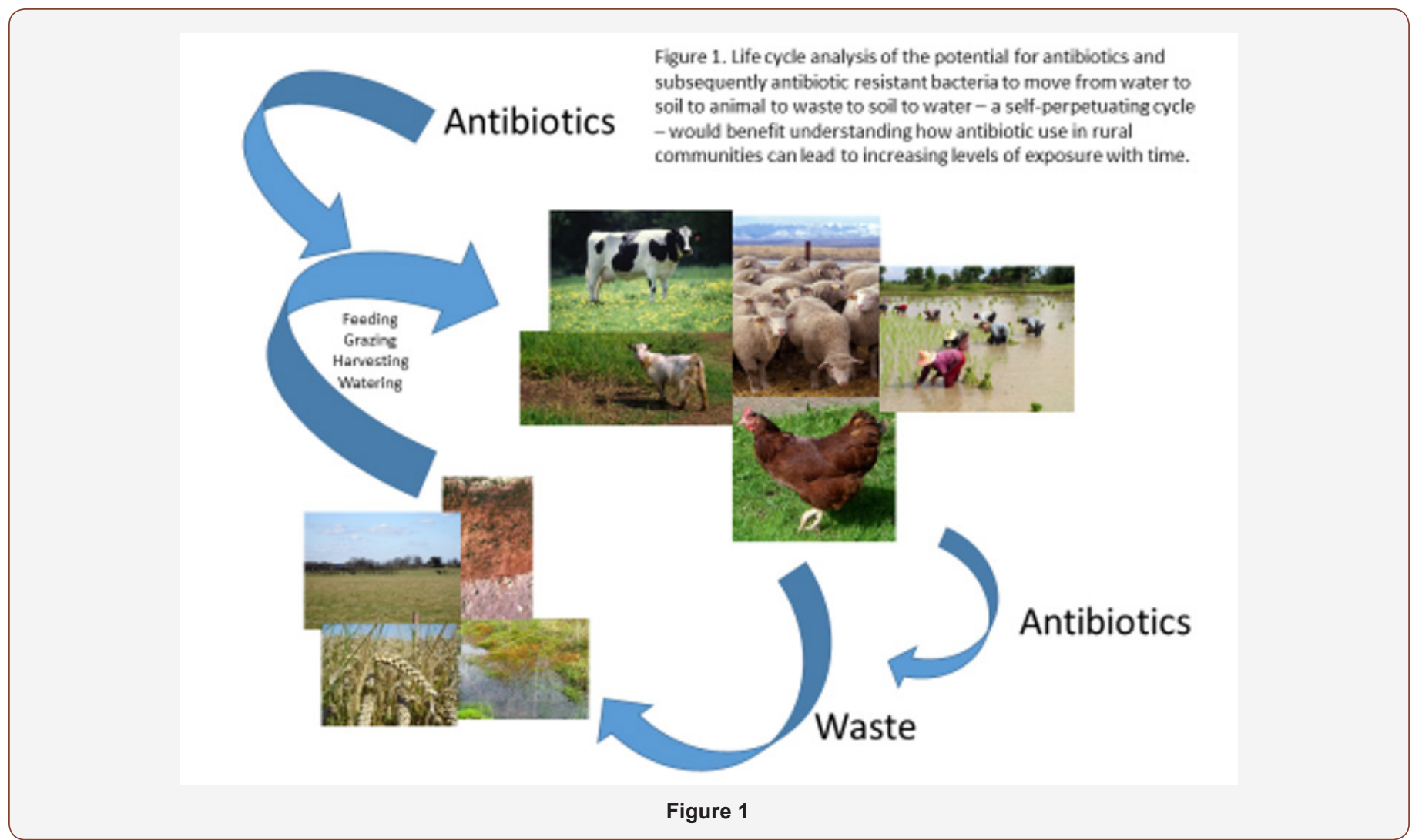

\section{Exposure routes}

Clinical overuse and self-prescription of medicines including antibiotics is common in developing countries. The most common sink of antibiotics includes the sewage and urban environments that may contaminate shallow- and ground-water. These sources may harbor resistant bacteria released from hospitals and houses that are transferred from patients to the general population living in close proximity or sharing a common environment. Once released in the environment, antibiotics can persist for long periods in these sinks to become a potential source of chronic exposure for aquatic species and pathogens. Aquatic ecosystems are a major reservoir for antibiotic resistant bacteria. Antibiotic resistant bacteria in water can act as a carrier and facilitate the spread of antibiotic resistance [10].

High concentrations of antibiotics and large numbers of resistant bacteria carried by runoff and wastewater can find their 
way into rural areas. In aquaculture, the direct use of antibiotics in water is very common [11]. Antibiotic residues may persist for long periods in water resulting in resistance of aquatic organisms [12] triggering further resistance in various directions by becoming a part of the environmental and human food chain [13].

Shallow rural aquifers not only receive antibiotics from urban environments but the practice of using antibiotics as food additives and therapeutic dosages given to livestock are also contributing sources. Consequently, noxious components of antibiotic use both in parent compound, metabolites, and in the form of resistant bacteria eventually end up in rural settings. Because rural environments differ from urban environments (being highly diverse) the human influence becomes extremely important when one moves outside cities.

In this context, pharmaceutical units, mostly located at the urban-rural fringe, are noticeable as their surrounding environment constantly receives antibiotics (though diluted) from such factories. Antibiotics build up in soil and promote the intermingling of ubiquitous soil microorganisms with antibiotic-producing bacteria, which influences indigenous bacteria to become resistant. This process triggers acute and chronic exposure. In the former case, plants and soil are affected mainly by antibiotic residuals and exposed microorganisms, respectively. Chronic exposure is intricate because it reflects processes occurring with time following soil contamination, such as adsorption of antibiotics in soil carried either by water, leachates, irrigation water, or runoff from fields. In this case, the long-term persistence of antibiotics depends on soil characteristics, especially the presence of clay minerals and organic colloids, and also the physico-chemical nature of the drug itself, which determines the rate of adsorption in soil and its ease of decomposition.

It is noteworthy that soil and water receiving a mixture of antibiotics in sub-lethal concentrations, or below regulatory limits, is usually considered safe [14]. However, the fact which is ignored on most occasions is that constant addition of various antibiotics in soil and water at low levels could saturate clay and colloid adsorption sites and will undoubtedly cause synergistic effects on indigenous microorganisms that are largely unknown. Ultimately, a condition can be reached where the total concentration of antibiotic mixtures exceeds threshold levels sufficient to cause harm to exposed organisms - including humans.

The impact of changing resistance among bacteria is perceived as a threat because of genetic modification triggered when these bacteria are exposed to antibiotics for a period of indefinite length or eliminating certain groups of microorganisms. For instance, bacterial populations susceptible to high doses of antibiotics, leave behind those organisms showing the least adaptive capacity to the prevailing soil environment and limited in performing supportive functional roles in soil as well as in modifying the rhizosphere microbial community [15]. This is also true in the human gut, where antibiotic exposure impairs the capacity of gut microflora to ferment carbohydrates, leading (for instance) to diarrhea [16].
In the backdrop to climate change scenarios, in which water scarcity is an imminent challenge, the use of treated wastewater for agricultural needs is common [17]. Irrigation using treated wastewater has solved water shortage problems in many areas. However, dangers associated with this practice also potentially threaten the environment and public health. Microorganisms are highly susceptible biological entities inhabiting agricultural soil that is irrigated with treated wastewater on a long-term basis. The development of antibiotic resistant microbes and antibiotic resistance genes depends on the properties of native soil. A high organic content and soils rich in clay may promote adsorption of compounds released from antibiotics - it does not imply degradation. This phenomenon is less emphasized in literature as being a potential source of exposure, especially when any change in soil reaction such as $\mathrm{pH}$ fluctuation, temperature and soil moisture changes, or root exudates can shift the adsorption/absorption equilibrium to any direction, thus influencing antibiotic persistence in soil.

Resistant bacteria from animals that are shed into soil and ground water specifically contaminate agriculture workers, who could then spread these microbes through human networks by means of fomites and direct contact and tainted meat through the butchering procedure. Testing of retail meat items in stores reliably uncovers high rates of Enterobacteriaceae in chicken, turkey, pork, and hamburger [18]. A disturbing number of these microorganisms are antibiotic resistant, and when the meat is handled before cooking or incompletely cooked meat is ingested, it is possible to ingest the antibiotic resistant microbes. The U.S. Center for Disease Control and Prevention has assessed that this course of transmission represents 20 percent of the antibiotic resistant contamination in people [19]. The real rate may well be larger even before representing the ecological spread of resistant bacteria because it is difficult to represent the extra adjustment of human-to-human transmission after the underlying presentation of resistant bacteria from animals to people.

Antibiotic resistance is a natural phenomenon and it has been identified through metagenomic-based analysis of DNA from 30,000 year old North Canadian permafrost sediments [20]. A vancomycin resistance element in the sediments was identified that is structurally and functionally similar to the gene vanA recognized in modern clinical pathogens. The need today is to study native antibiotic resistance present in the soil microbiome, but this phenomenon is neglected. A better understanding of bacterial diversity and ecology is still required for antibiotic resistance transfer through bacteria. The question is still unanswered about the modes by which antibiotic resistance is transmitted in water, which may be relevant for human health. The human microbiome determines the characteristics of the bacteria that may colonize the human body. In the future, this brings discussion of antibiotic resistance transmission from water to humans and from humans to water.

Another exposure route is consumption of vegetables and fruits irrigated with treated and untreated wastewater. Plant material 
containing antibiotics consumed by humans may give resistance to human pathogens and can be a genuine risk. This expanding resistance might be of concern both for human and animals if antibiotics are found in food crops.

Multiple routes of soil and water contamination by ethical and unethical antibiotic use demands multiple routes of investigation (Figure 2). What is the amount and characteristics of the antibiotic land being received by soil and water? To what extent do these antibiotics persist in active form? To what extent do individual antibiotics, collections of antibiotics, and classes of antibiotics contribute to acute and chronic exposure and the creation of higher levels of intrinsic, acquired, and developed resistance? Does that exposure change critical soil function or resistance and resilience in the microbial community? Does the persistence of antibiotics in natural reservoirs enhance human exposure to chronic sublethal concentrations of antibiotics, or a continual exposure to microorganisms that have themselves developed resistance?

\section{Figure 2. A conceptual diagram of research needs necessary to evaluate the risks associated with chronic exposure to antibiotics,}

\section{Re-use/Recycling}

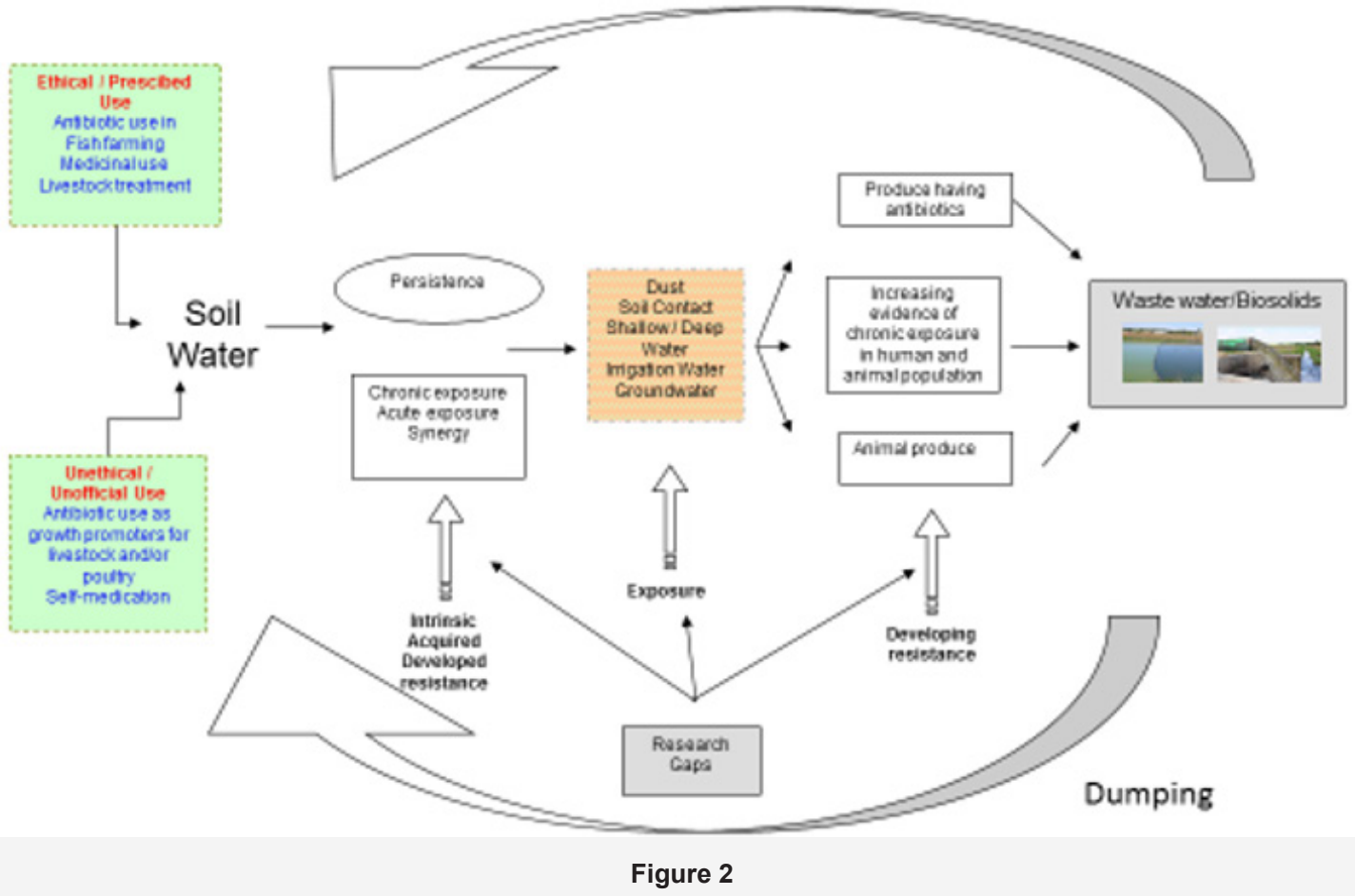

\section{Human Exposure and Epidemiology in Rural} Communities

Demonstrating evidence of antibiotic exposure is essential to propose intervention strategies. Here we argue the necessity to design exclusive studies able to identify unexplored antibiotic exposure pathways that are responsible for developing multiple effects on exposed microbial and human populations. There is a research gap in terms of defining the extent of exposure when antibiotics are released in high concentration and enter water systems that are not used for irrigation. This is in contrast to a situation in which there is more intense exposure due to constant addition of sub-lethal levels of antibiotics in environments (especially in rural areas) where the human population by virtue of its occupation or residential location is exposed through multiple routes such as soil dust (aerosols), direct soil contact, shallow/deep water, and irrigation or ground water that may contain antibiotics (Figure 2). Mc Donald, et al. [21] and Rothschild, et al. [22] both recently reported that occupation and environmental factors rather than ethnic origin or ancestry were the key determinants to characterizing human gut microbiome diversity. Studies are therefore required to answer the question: is there sufficient evidence of antibiotic presence in samples collected from diverse compartments of the rural environment? Once this research gap is appropriately addressed (i.e. confirmation or existence of chronic exposure) the next logical direction would be to differentiate populations according to their susceptibility.

Here, only in-depth epidemiological findings can demonstrate the effect of chronic exposure. The imminent challenge to perform such research would be examining the resistant microorganisms present in environmental media (soil, aerosols, and water, etc.) and categorizing them as either pathogenic or non-pathogenic. Part of the microbial community on ingested foods and in water becomes a part of the human microbiome through engraftment [23]. Upon entry in human body, both have implications, as people may develop antibiotic resistance in the case of the former whereas transfer of resistance is the most probable outcome in the latter case. 


\section{Probable epidemiological outcomes}

The risk associated with the antibiotics to human health is expressed three ways: 1) antibiotic reaction; 2) prevalence of resistant bacteria that are of clinical importance; 3) disruption of normal function in the human gut microflora. One of the antibiotic reactions, due to consumption, is allergic reaction, a kind of adverse drug reaction. Another type of reaction is chronic toxicity, in which antibiotics accumulate in the human body. The quinolones and tetracyclines, for example, influence the development of infant teeth and chloramphenicol may cause aplastic anemia and agranulocytosis. Some antibiotics can be metabolized in the human body and metabolites may be more toxic than the parent compound. If antibiotic-contaminated food and water is used it can result in chronic toxicity.

Water acts as a reservoir of antibacterial genes and is the origin of resistance genes that are already released to the environment and acquired by human pathogens. Water facilitates exchange of antibiotic resistance genes between pathogenic and nonpathogenic bacteria [24]. The abundance of bacteria in humans plays an important role in the transfer of antibiotic resistance to human pathogens. This means the human microbiome acts as an intermediate resistance reservoir [25]. In the absence of control by the normal human gut microflora pathogens such as Clostridium dificile, Clostridium perfringens, and Klebsiella oxytoca, can manifest themselves [16], and their persistence is enhanced by acquisition of antibiotic resistance.

Age is of great importance for exposure to antibiotics. The gut flora plays a key role in metabolic function such as fermentation and energy salvage, homeostasis of the immune system, and protection against pathogens [26]. Antibiotics at low levels increase the capacity of ingested microbes to colonize the human gut by disrupting the resistance of indigenous microbes to colonization [27]. This is of consequence for infants developing their microflora, as early exposure to antibiotics can alter the gut microbiome and subsequently potential for adverse health [28].

To better comprehend the public exposure to antibiotics in the environment, we need to understand the gradient that exists in terms of simple distance from human settlements, or of specific means of "extending" human influence, such as along water streams, or even within birds. Antibiotics and/or antibiotic resistant bacteria can be found across these gradients, establishing gradients of their own, which can help pinpoint their origin; non-antibiotic selective or maintenance pressures must also be considered along with other human influences.

Of particular interest are three scenarios, that place copious amounts of antibiotics in rural environments: (1) the release of antibiotics from drug manufacturing factories (often in industrial areas, but outside of cities) usually through wastewater dumped into water bodies; (2) the agricultural use of antibiotics, to treat or prevent infections in animals or plants, or as "growth promoters;" this form of use, in addition to releasing antibiotics, also selects for resistant bacteria, that are released to the environment, sometimes in huge amounts, as when manure is used as fertilizer; (3) it would be naïve to suppose that rural areas are only the 'sink' of antibiotics and their residues. What must be taken into account is, in resource limited settings, that rural areas are potential sources of treated and untreated wastewater comprising human and animal waste as well as biosolids that may directly contribute to the water supply system that eventually is used for cropping and irrigation. Life cycle analysis of the potential for antibiotics and subsequently antibiotic resistant bacteria to move from water to soil to animal to waste to soil to water - a self-perpetuating cycle - would benefit understanding how antibiotic use in rural communities can lead to increasing levels of exposure with time.

\section{Conclusion}

The negative effects on public health due to chronic exposure to sub-lethal concentrations of antibiotics is not recognized as a research crisis. Global efforts by WHO to return to a pre-antibiotic era have made it obligatory for member nations to ratify a global action plan for antimicrobials. In this context, identifying exposure routes and susceptible population is of prime significance. Specific research approaches are needed to understand the nexus between ease of antibiotic release, anticipated human exposure, and uninvestigated exposure routes in rural areas. We conclude that occupational groups in farmyards, rural populations living in the vicinity of industrial zones, wastewater irrigation fields, and produce from animals and plants containing antibiotics are potential determinant factors that need to be simultaneously analyzed to present a holistic view of antibiotic resistance scenarios. Environments carrying resistance derived from antibiotics in various studies have reported intrinsic resistance as a key "contaminant". However, such studies are rarely multi-disciplinary, consequently, several key determinants of exposure are overlooked. To avoid errors in methodological protocols, investigative procedures need to be rationalized to present more convincing evidence for exposure pathways, public health outcomes, and pertinent interventions to reduce antibiotic resistance.

\section{Acknowledgement}

None.

\section{Conflict of Interest}

No conflict of interest.

\section{References}

1. Van Boeckel TP, Brower C, Gilbert M, Grenfell BT, Levin SA, et al. (2015) Global trends in antimicrobial use in food animals. Proceedings of the National Academy of Sciences 112: 5649-5654.

2. Van Boeckel TP, Gandra S, Ashok A, Caudron Q, Grenfell BT, et al. (2014) Global antibiotic consumption 2000 to 2010: an analysis of national pharmaceutical sales data. The Lancet Infectious Diseases 14: 742-750.

3. Klein EY, TP Van Boeckel, EM Martinez, S Pant, S Gandra, S, et al. (2018) Global increase and geographic convergence in antibiotic consumption between 2000 and 2015. Proceedings of the National Academy of Sciences 115(15): E3463-E3470.

4. Brandt KK, Amézquita A, Backhaus T, Boxall A, Coors, et al. (2015) Ecotoxicological assessment of antibiotics: a call for improved consideration of microorganisms. Environment International 85: 189205 . 
5. Monsalvo VM (Ed.) (2015) Wastewater and Public Health: Bacterial and Pharmaceutical Exposures. CRC Press, Boca Raton FL.

6. Kümmerer K (2009) Antibiotics in the aquatic environment-a reviewpart I. Chemosphere 75: 417-434.

7. Pan M, LM Chu (2016) Adsorption and degradation of five selected antibiotics in agricultural soil. Science of the Total Environment 545: 48-56.

8. Yang Q Wang R, Ren S, Szoboszlay M, Moe LA, et al. (2016) Practical survey on antibiotic-resistant bacterial communities in livestock manure and manure-amended soil. Journal of Environmental Science and Health, PartB 51: 14-23.

9. Zhang YL, Lin SS, Dai CM, Shi L, Zhou XF, et al. (2014) Sorptiondesorption and transport of trimethoprim and sulfonamide antibiotics in agricultural soil: effect of soil type, dissolved organic matter, and $\mathrm{pH}$. Environmental Science and Pollution Research 21: 5827-5835.

10. Riaz L, Mahmood T, Khalid A, Rashid A, Ahmed Siddique MB, et al. (2018) Fluoroquinolones (FQs) in the environment: a review on their abundance, sorption and toxicity in soil. Chemosphere 191: 704-720.

11. Riaz L, Mahmood T, Kamal A, Shafqat M, Rashid A, et al. (2017) Industria release of fluoroquinolones (FQs) in the waste water bodies with their associated ecological risk in Pakistan. Environmental Toxicology and Pharmacolog 52: 14-20.

12. Taylor NG, Verner Jeffreys DW, Baker Austin C (2011) Aquatic systems: maintaining, mixing and mobilising antimicrobial resistance? Trends in Ecology \& Evolution 26: 278-284.

13. Tamminen M, Karkman A, Lõhmus A, Muziasari WI, Takasu H, et al. (2010) Tetracycline resistance genes persist at aquaculture farms in the absence of selection pressure. Environmental Science \& Technology 45 386-391.

14. Bengtsson Palme J, DGJ Larsson (2016) Concentrations of antibiotics predicted to select for resistant bacteria: Proposed limits for environmental regulation. Environment International 86: 140-149.

15. Banerjee S, ED Angelo (2013) Livestock antibiotic effects on nitrification, denitrification, and microbial community composition in soils. Open Journal of Soil Science 3: 203-212.

16. Beaugerie L, JC Petit (2004) Antibiotic-associated diarrhoea. Best Practice \& Research Clinical Gastroenterology 18: 337-52.
17. Yusuf S (2017) Pakistan among 5 countries that account for $90 \%$ of wastewater irrigated farms, study finds. MIT Technology Review Pakistan.

18. Elliott K (2015) Antibiotics on the farm: Agriculture's role in drug resistance. CGD Policy, p. 59.

19. Spellberg B, GR Hansen, A Kar, CD Cordova, LB Price, et al. (2016) Antibiotic resistance in humans and animals. National Academy of Medicine, p. 15

20. Gatica J, E Cytryn (2013) Impact of treated wastewater irrigation on antibiotic resistance in the soil microbiome. Environmental Science and Pollution Research 20: 3529-3538.

21. Mc Donald D, Hyde E, Debelius JW, Morton JT, Gonzalez A, et al. (2018) American Gut: An open platform for citizen science microbiome research. mSystems 3(3): e00031-18.

22. Daphna Rothschild, Omer Weissbrod, Elad Barkan, Tal Korem, David Zeevi, et al. (2018) Environment dominates over host genetics in shaping human gut microbiota.

23. Shepherd ES, WS DeLoache, KM Pruss, Weston R Whitaker, Justin L Sonnenburg, et al. (2018) An exclusive metabolic niche enables strain engraftment in the gut microbiota. Nature 557: 434-438.

24. Rizzo L, Manaia C, Merlin C, Schwartz T, Dagot C, et al. (2013) Urban wastewater treatment plants as hotspots for antibiotic resistant bacteria and genes spread into the environment: a review. Science of the Total Environment 447: 345-360.

25. Forslund K, Sunagawa S, Kultima JR, Mende DR, Arumugam M, et al. (2013) Country-specific antibiotic use practices impact the human gut resistome. Genome Research 23(7): 1163-1169.

26. Guarner F, JR Malagelada (2003) Gut flora in health and disease. The Lancet 361: 512-519.

27. Carman RJ, Simon MA, Fernández H, Miller MA, Bartholomew MJ, et al (2004) Ciprofloxacin at low levels disrupts colonization resistance of human fecal microflora growing in chemostats. Regulatory Toxicology and Pharmacology 40: 319-26.

28. Cho I, Yamanishi S, Cox L, Methé BA, Zavadil J, et al. (2012) Antibiotics in early life alter the murine colonic microbiome and adiposity. Nature 488: 621-626. 